\title{
LA GESTIÓN DEL RIESGO DE DESASTRES EN EL PERÚ
}

\author{
DISASTER RISK MANAGEMENT IN PERU
}

Arturo Isla Zevallos

\section{Resumen}

En el Perú existen una serie de fenómenos o peligros recurrentes, naturales o antrópicos, que causan un enorme impacto en la vida, y en los ámbitos sociales, económicos y ambientales del país. La estadística muestra que en los últimos años, las emergencias y los daños que causan son cada vez más de traumáticas consecuencias. Ante esta evidencia, la respuesta de la sociedad presenta una asombrosa debilidad. En particular, el liderazgo del Sistema Nacional de Gestión del Riesgo de Desastres es apenas perceptible y, todo indica que el involucramiento efectivo de los más de dos mil entes de gobierno, a nivel nacional, regional y local, para estimar, prevenir y reducir el riesgo de desastres es todavía un tema de largo plazo.

Palabras clave: Peligros; riesgo de desastres; emergencias, gestión del riesgo de desastres, impactos de fenómenos

\section{Abstract}

In Peru there are a series of recurrent phenomena or dangers, natural or anthropic, that cause an enormous impact on life, and in the social, economic and environmental spheres of the country. Statistics show that in recent years, emergencies and the damage they cause are increasingly traumatic consequences. Given this evidence, the response of society shows an amazing weakness. In particular, the leadership of the National Disaster Risk Management System is 
barely perceptible and, all indications are that the effective involvement of the more than two thousand government entities, at national, regional and local levels, to estimate, prevent and reduce the Disaster risk is still a long-term issue.

Key words: Dangers: risk of disasters; emergencies; disaster risk management; phenomenon impacts.

"Y asi ha habido otros muy muchos milagros y castigos en el tiempo del Ynga. No se escribe sino son los testigos de la caida de los cerros y peñas derrumbadas. Y asi se escribe toda la suma; por eso el castigo de Dios le llaman pachacuti [el que transforma la tierra], pacha ticra [el que la pone cabeza abajo]". Guamán Poma, Nueva crónica y bUen Gobierno (1615)

"Viene corriendo El Niño desde la isla de Pascua, tibio y malsano el infante de la muerte por agua, azotado contra las costas del Perú, sofocando en su abrazo caliente las anchoas y las algas [...] pesado y sudoroso nada. El Niño arrojando peces contra las paredes del continente, adormeciéndolo y pudriéndolo todo [...] los vientos enloquecidos y desplazados”. Carlos Fuentes, citado Por Fagan (2010)

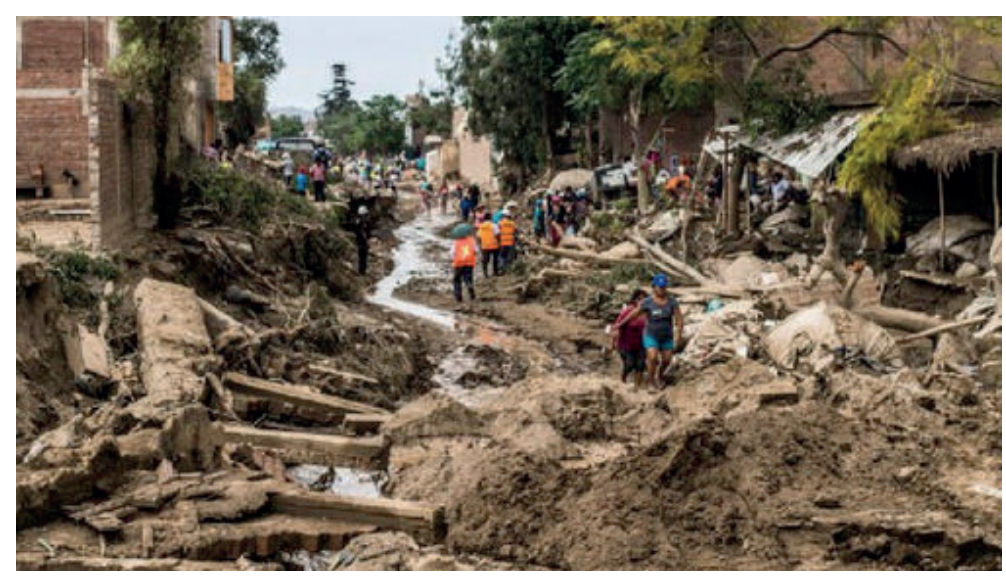




\section{LOS RIESGOS DE DESASTRES}

\subsection{Las emergencias en el Perú}

El Perú presenta una simetría esplendorosa: "Recursos por un lado y recursos por el otro lado". Pero bien es sabido que los recursos en exceso originan problemas. Por ejemplo, las lluvias exageradas causan inundaciones y huaycos, los que a su vez tienen efectos en viviendas, edificaciones de servicios públicos y de empresas, obras de infraestructura, campos de cultivo, entre muchos daños, así como en la vida misma de las personas.

Este espectáculo continuo de desastres ocurridos que dibujan el lienzo del territorio peruano reconfigurándolo dolorosamente, permite aseverar la existencia de otra simetría, esta vez horrorosa: "desastres de un lado y desastres por el otro lado". E1 registro histórico de las emergencias habidas en el país (INDECI, 2017), constata lo expresado (Cuadros $\mathrm{N}^{\circ} 1 \mathrm{y}$ Cuadro $\left.N^{\circ} 2\right)$. Son emergencias en número significativo y cuya tendencia es errática. Las cifras no nos enseñan un parámetro definido; por ejemplo, las emergencias en el 2014 fueron menores que en el 2013, no obstante, el número de muertos aumentó; aná- logamente, los daños a las viviendas se redujeron, pero la superficie agropecuaria afectada se multiplicó en seis veces. Esto quiere decir que en algunos años los fenómenos afectan más a las áreas urbanas, mientras que en otros son las áreas rurales las más sensibles; aunque también sucede en ambas.

El cuadro $\mathrm{N}^{\circ} 1$ referido a los últimos 14 años (2003 al 2016) nos muestra que han ocurrido un promedio anual alrededor de 4 mil emergencias, y que el pico de la estadística en cuanto a número de emergencias se encuentra en el 2016 con 5,167 registros. En cuanto a daños, es el año 2007 el que registra mayores afectaciones personales y en viviendas, debido al sismo de Pisco en el departamento de Ica, el cual impactó en otros departamentos del sur del país. Análogamente, el año 2012 nos ofrece los daños significativos causados por las inundaciones en el departamento de San Martín y Loreto, así como de heladas en la sierra y de friaje en la selva en varios departamentos del Perú; además, de presentar una cifra que supera el millón y medio de personas como afectadas y el mayor número de daños en la agricultura, esto debido a las bajas temperaturas y sequías. 


\section{CUADRO N $^{\circ}$ 1: EMERGENCIAS Y DAÑOS A NIVEL NACIONAL: 2003-2014}

\begin{tabular}{|c|c|c|c|c|c|c|c|c|c|c|}
\hline \multirow{2}{*}{ AÑo } & \multirow{2}{*}{ EMER } & \multicolumn{5}{|c|}{ DANNOS PERSONALES } & \multicolumn{2}{|c|}{$\begin{array}{l}\text { DANOOS EN } \\
\text { VIVIENDAS }\end{array}$} & \multicolumn{2}{|c|}{$\begin{array}{c}\text { DANOOS EN HAS DE } \\
\text { CULTIVO }\end{array}$} \\
\hline & & FALLEC & LESIONAD & DESAPAR & DAMNIF & AFECT & AFECT & DESTR & AFECT & PERDIDOS \\
\hline TOTAL & 61,856 & 2,457 & 8,448 & 346 & $1,635,567$ & $14,314,771$ & $1,049,390$ & 210,797 & $1,795,191$ & $1,042,249$ \\
\hline 2003 & 3,316 & 213 & 373 & 30 & 62,347 & 246,159 & 34,679 & 8,525 & 36,688 & 13,615 \\
\hline 2004 & 4,038 & 144 & 284 & 28 & 45,947 & 919,895 & 31,980 & 6,108 & 253,058 & 90,265 \\
\hline 2005 & 4,773 & 122 & 370 & 17 & 75,658 & 876,599 & 30,198 & 9,090 & 192,558 & 14,804 \\
\hline 2006 & 4,495 & 88 & 260 & 6 & 31,046 & 997,337 & 53,340 & 5,566 & 75,973 & 8,718 \\
\hline 2007 & 4,536 & 701 & 2,436 & 11 & 496,263 & $1,662,413$ & 65,294 & 96,357 & 32,310 & 5,719 \\
\hline 2008 & 4,545 & 165 & 273 & 34 & 84,410 & $1,368,056$ & 151,794 & 15,543 & 103,588 & 18,098 \\
\hline 2009 & 4,037 & 112 & 226 & 59 & 31,578 & 942,279 & 62,461 & 6,624 & 6,375 & 276 \\
\hline 2010 & 4,535 & 131 & 2,491 & 53 & 74,382 & 834,487 & 44,595 & 12,262 & 31,289 & 5,636 \\
\hline 2011 & 4,816 & 119 & 262 & 19 & 177,673 & 897,974 & 101,293 & 20,954 & 70,246 & 24,399 \\
\hline 2012 & 5,127 & 179 & 377 & 31 & 333,367 & 865,800 & 166,606 & 12,871 & 47,859 & 45,514 \\
\hline 2013 & 4,379 & 136 & 164 & 18 & 52,902 & 724,219 & 58,843 & 4,420 & 35,473 & 12,948 \\
\hline 2014 & 3,770 & 73 & 123 & 17 & 30,485 & 309,539 & 33,418 & 2,624 & 214,611 & 19,692 \\
\hline 2015 & 4,322 & 171 & 474 & 18 & 64,920 & $1,427,432$ & 148,870 & 5,878 & 191,106 & 25,311 \\
\hline 2016 & 5,167 & 103 & 335 & 5 & 74,589 & $2,242,582$ & 66,019 & 3,975 & 504,057 & 757,254 \\
\hline
\end{tabular}

Fuente : COEN-SINPAD-INDECI

Elaboración : Sub Dirección de Aplicaciones Estadisticas - DIPPE - INDECI

\section{CUADRO N 2: TIPOS DE DAÑOS POR FENÓMENO: 2016}

\begin{tabular}{|c|c|c|c|c|c|c|c|c|c|c|}
\hline \multirow{3}{*}{ FENOMENO } & \multirow{3}{*}{ EMER } & \multicolumn{5}{|c|}{ DAÑOS PERSONALES } & \multicolumn{4}{|c|}{ DAÑOS MATERIALES } \\
\hline & & \multirow[b]{2}{*}{ DAMNIF } & \multirow[b]{2}{*}{ AFECT } & \multirow[b]{2}{*}{ DESAP } & \multirow[b]{2}{*}{ LESION } & \multirow[b]{2}{*}{ FALLEC } & \multicolumn{2}{|c|}{ VIVIENDAS } & \multicolumn{2}{|c|}{ HAS CULTIVO } \\
\hline & & & & & & & DESTR & AFECT & PERD & AFECT \\
\hline TOTAL & 5,167 & 74,589 & $2,242,582$ & 5 & 335 & 103 & 3,975 & 66,019 & 757,254 & 504,057 \\
\hline ALUD & 9 & 47 & 616 & 0 & 0 & 0 & 4 & 86 & 0 & $\overline{10}$ \\
\hline BAJAS TEMPERATURAS & 1,205 & 6,565 & $1,278,907$ & 0 & 4 & 9 & 45 & 7,625 & 47,380 & 216,129 \\
\hline CONTAMINACIÓN & 5 & 40 & 620 & 0 & 4 & 0 & 이 & 4 & 0 & \\
\hline DERRAME DE SUSTANCIAA & 16 & 14 & 16,711 & 0 & 3 & 6 & 3 & 18 & 0 & 16 \\
\hline DERRUMBE & 39 & 67 & 75 & 0) & 12 & 3 & 7 & 22 & 0 & 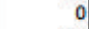 \\
\hline DESLIZAMIENTO & 64 & 256 & 2,916 & 1 & 9 & 4 & 15 & 685 & 35 & 16 \\
\hline EPIDEMIAS & 17 & 0 & 27,643 & 0 & 0 & 이 & 이 & 0 & 0 & 7 \\
\hline EROSIÓN & 23 & 328 & 1,345 & 0 & 0 & 0 & 52 & 342 & 0 & \\
\hline EXPLOSIÓN & 3 & 0 & 3 & 0 & 14 & 8 & 이 & 1 & 0 & 0 \\
\hline HUAYCO & 83 & 624 & 5,429 & 0 & 24 & 7 & 56 & 921 & 73 & 177 \\
\hline IINCENDIO FORESTAL & 281 & 288 & 44,063 & 이 & 34 & 2 & 23 & 3,533 & 2,995 & 925 \\
\hline INCENDIO URB. E INDUST. & 852 & 6,388 & 2,204 & 이 & 72 & 23 & 1,014 & 403 & 2 & 2 \\
\hline INUNDACIÓN & 128 & 2,370 & 67,365 & 0 & 3 & 1 & 263 & 14,454 & 3,267 & 5,881 \\
\hline LLUVIA INTENSA & 755 & 8,807 & 118,100 & 1 & 5 & 10 & 1,404 & 25,308 & 645 & 2,823 \\
\hline MAREJADA & 7 & 0 & 750 & 0 & 0 & 0 & 이 & 0 & 152 & 3,767 \\
\hline PLAGAS & 13 & 0 & 22,124 & 0 & 0 & 0 & 이 & 1,014 & 0 & \\
\hline SEQUIA & 850 & 32,634 & 271,061 & 0 & 0 & 이 & 이 & 0 & 698,831 & 255,659 \\
\hline SISMO & 56 & 7,892 & 15,824 & 0] & 81 & 9] & 667 & 4,619 & 2 & 2,152 \\
\hline TORMENTA ELECTRICA & 25 & 74 & 20 & 0 & 0 & 8 & 2 & 4 & 0 & \\
\hline VIENTOS FUERTES & 702 & 8,140 & 25,892 & 3 & 66 & 7 & 407 & 6,203 & 3,366 & 2,260 \\
\hline OTROS & 34 & 55 & 340,914 & 의 & 4 & 6 & 13 & 777 & 506 & 14,233 \\
\hline
\end{tabular}

Fuente : SINPAD-COEN-INDECI

Elaboración : Sub-Dirección de Aplicaciones Estadisticas - DIPPE - INDECI

"Los fenómenos que han ocurrido a nivel nacional en mayor número durante el año 2016 y que han ocasionado daños son las Bajas Temperaturas con un total de registros de 1,205 eventos, alcanzando el $23.32 \%$, le sigue en mayoria de ocurrencia los Incendios Urbanos e Industriales con un total de 852 
registros alcanzando el $16.48 \%$ del total anual. Le sigue luego, las Sequías, las Lluvias Intensas y los Vientos Fuertes". (INDECI, 2017).

Aunque todavía no se tiene la estadística completa del 2017, este año en curso, las inundaciones habidas a comienzos de año han gatillado tanto las emergencias como los daños ocurridos, según el cuadro $\mathrm{N}^{\circ} 3$ con información del Sistema de Información Nacional para la Respuesta y la Rehabilitación, SINPAD del INDECI.

\section{CUADRO $N^{\circ}$ 3: EMERGENCIAS Y DAÑOS TOTALES: ENERO-OCTUBRE 2017}

\begin{tabular}{|c|c|c|c|c|c|c|c|c|c|}
\hline MESES & EMERGENCIAS & FALLECIDOS & DESPARECIDOS & HERIDOS & DAMNIFICADOS & AFECTADOS & $\begin{array}{c}\text { VIVIENDAS } \\
\text { AFECTADAS }\end{array}$ & \begin{tabular}{|c|} 
VIVIENDAS \\
DESTRUIDAS
\end{tabular} & $\begin{array}{c}\text { Ha. DE } \\
\text { CULTIVOS } \\
\text { DESTRUIDOS }\end{array}$ \\
\hline ENERO & 975 & 39 & 6 & 108 & 28,907 & 359,917 & 81,534 & 3,335 & 10,004 \\
\hline FEBRERO & 1,129 & 26 & 5 & 33 & 33,317 & 219,831 & 49,883 & 3,022 & 3,228 \\
\hline MARZO & 2,557 & 106 & 12 & 410 & 209,265 & 998,206 & 233,568 & 28,272 & 35,935 \\
\hline ABRIL & 661 & 12 & 0 & 13 & 6,458 & 61,783 & 10,255 & 838 & 1,364 \\
\hline MAYO & 325 & 0 & 0 & 13 & 11,926 & 205,495 & 11,673 & 2,364 & 1,553 \\
\hline JUNIO & 217 & 9 & 0 & 16 & 843 & 75,027 & 2,181 & 97 & 445 \\
\hline JULIO & 260 & 2 & 0 & 14 & 929 & 72,048 & 1,179 & 75 & 1,367 \\
\hline AGOSTO & 331 & 1 & 0 & 4 & 724 & 50,879 & 670 & 118 & 187 \\
\hline SETIEMBRE & 241 & 7 & 0 & 7 & 1,204 & 18,706 & 382 & 139 & 11 \\
\hline OCTUBRE & 248 & 2,649 & 217 & 140,810 & 339,324 & 842,518 & 59,957 & 41,189 & 330 \\
\hline NOVIEMBRE & 0 & 0 & 0 & 0 & 0 & 0 & 0 & 0 & 0 \\
\hline DICIEMBRE & 0 & 0 & 0 & 0 & 0 & 0 & 0 & 0 & 0 \\
\hline TOTALES & 6,944 & 2,851 & 240 & 141,428 & 632,897 & $2,904,410$ & 451,282 & 79,449 & 54,424 \\
\hline
\end{tabular}

FUENTE: SINPAD-INDECI, 2017

Al 07 de agosto del 2017, el último informe de INDECI de evaluación del Niño Costero en lo que va del año contabiliza en cuanto a la vida y la salud de las personas 162 fallecidos, 500 heridos, 19 desaparecidos, 285,955 damnificados y 1'559,487 afectados. En cuanto a daños en vivienda e institucionales, 66,093 viviendas destruidas e inhabitables, 371,370 viviendas afectadas, 354 instituciones educativas destruidas e inhabitables, 3,266 instituciones educativas afectadas, 64 establecimientos de salud destruidos e inhabitables y 1,044 establecimientos de salud afectados. Respecto a infraestructura social, 3,956 $\mathrm{km}$ de caminos rurales destruidos, $41,180 \mathrm{~km}$ de ca- minos rurales afectados, 4,391 $\mathrm{km}$ de carreteras destruidas, 11,761 carreteras afectadas, 489 puentes destruidos y 881 puentes afectados. En lo que corresponde al sector agricultura, el principal impactado en el ámbito rural, 50,154 Ha perdidas, 107,827 Ha de cultivo afectadas, 22,674 canales de riesgo destruidos y 49,479 canales de riesgo afectados. (INDECI, 2017)

\subsection{Conceptos básicos de la gestión del riesgo de desastres}

En el Perú, existen diversas condiciones climáticas que, actuando en conjunto con los factores meteorológicos, originan situaciones anormales 
en cada una de las regiones del territorio. Entre otros, puede mencionarse a la Corriente Oceánica Peruana de Humboldt, el Anticiclón del Pacífico Sur, la Cordillera de los Andes, la Corriente Ecuatorial Oceánica y las Zonas de Convergencia Intertropical (ZCIT).

Generalmente, los desastres que ocurren con mayor frecuencia son los huaycos e inundaciones producidos por las lluvias torrenciales del perio-

do húmedo entre enero y marzo, las que dan origen a los "huaycos" en las cuencas de las vertientes del $\mathrm{Pa}$ cífico y en las cuencas de la zona de la Sierra de la vertiente del Atlántico. La inundación es el evento que afecta significativamente la vida económica del país, debido a la falta de defensas ribereñas, especialmente en las zonas urbanas.

Además de la localización espacial inadecuada, se presentan patrones constructivos impropios, causando una suerte de fragilidad frente a riesgos. Adicionalmente, estas poblaciones, que recurrentemente afrontan situaciones críticas de desastres, tienen una limitada resiliencia para sobreponerse a situaciones adversas, un grado menor de adaptación y de actitud para el cambio, y un reducido aprendizaje de las experiencias vividas. Es común encontrar resistencias a la reubicación en pobladores asentados en quebradas y márgenes de ríos, en momentos inmediatos a haber sufrido un desastre.

En suma, la exposición, fragilidad y la falta de resiliencia, son aspectos de la vulnerabilidad. El nivel de riesgo está en función del nivel de peligro y el nivel de vulnerabilidad. La severidad del riesgo en cada lugar determinará la zonificación del riesgo con sus impactos probables para darle el tratamiento adecuado a cada zona, lo que implica la gestión del riesgo mediante medidas de prevención y reducción del riesgo. (Isla, 2015).

Las inundaciones se producen cuando las lluvias intensas o continuas, deshielos, oleajes, fallas en sistemas o estructuras hidráulicas, cambios en los cauces de ríos, desbordamiento de lagos, sobrepasan la capacidad de campo del suelo, o el volumen máximo de transporte del río o del lago es superado y el cauce principal se desborda e inunda los terrenos circundantes. Las inundaciones se caracterizan porque las aguas cubren grandes extensiones de tierra cuando estas poseen escasa o ninguna pendiente, y cuyos drenajes naturales son pequeños. En estos casos, las aguas que se van concentrando permanecen dias, semanas y hasta meses estancadas, pudiendo también aumentar sus volúmenes por nuevas precipitaciones pluviales. Las inundaciones pueden clasificarse por su duración y por su origen.

Por su duración, las inundaciones pueden ser dinámicas (rápidas) o estáticas (lentas). Las inundaciones rápidas se producen por efecto de lluvias intensas sobre rios cuyas cuencas presentan fuertes pendientes. Las crecidas de los rios son repentinas y de corta duración. Son las que 
producen los mayores daños en la población y la infraestructura que ocupan, debido a que el tiempo de reacción es casi nulo; por ejemplo, en los casos de los ríos de la cuenca del Océano Pacífico (La Leche, Tumbes, Rímac, Pisco, Ica, etc.).

Las inundaciones lentas se producen cuando las lluvias son persistentes y generalizadas, provocando un aumento paulatino del caudal del río hasta superar su capacidad máxima de transporte, por lo que el río se desborda, inundando áreas planas cercanas al mismo. A estas áreas se les denomina llanuras de inundación, como son, por ejemplo, los ríos de la selva baja y los de las parte bajas de las cuencas del pacífico.

Por su origen, las inundaciones pueden ser pluviales, fluviales o lacustres, o por tsunamis. Las inundaciones pluviales se producen por la acumulación de agua de lluvia en un determinado lugar o área geográfica, sin que este fenómeno coincida necesariamente con el desbordamiento de un cauce fluvial. Este tipo de inundación se genera tras un régimen de lluvias intensas, persistentes, es decir, por la concentración de un elevado volumen de lluvia en un intervalo de tiempo muy breve, o por la incidencia de una precipitación moderada y persistente durante un amplio período de tiempo sobre un suelo poco permeable. Las inundaciones fluviales son causadas por el desbordamiento de los ríos y los arroyos. Es atribuida al aumento brusco del volumen de agua más allá de lo que un lecho o cauce es capaz de transportar sin desbordarse durante lo que se denomina crecida, como consecuencia del exceso de lluvias. Las inundaciones lacustres son originadas por desbordamiento de lagos. Las inundaciones por tsunamis, o marinas, son producidas por un tsunami, el cual produce olas crecientes en altura conforme se acercan al litoral. Esta inundación es costera y afecta a las poblaciones establecidas en zonas bajas cercanas al nivel del mar.

\section{LOS RIESGOS DE DESASTRES}

\subsection{La gestión del riesgo de desastre}

Según la Ley N²9664, la gestión del riesgo de desastres "Es un proceso social cuyo fin último es la prevención, la reducción y el control permanente de los factores de riesgo de desastres en la sociedad, así como la adecuada preparación y respuesta ante situaciones de desastre, considerando las politicas nacionales con especial énfasis en aquellas relativas a materia económica, ambiental, de seguridad, defensa nacional y territorial de manera sostenible. La gestión del riesgo de desastres está basada en la investigación científica y de registro de informaciones, y orienta las políticas, estrategias y acciones en todos los niveles de gobierno y de la sociedad con la finalidad de proteger la vida de la población y el patrimonio de las personas y el Estado". (Congreso, 2011)

La gestión del riesgo de desastres se resume en las siguientes fases 
(Gráfico $N^{\circ} 1$ ): Estimación del riesgo que se estima en función del nivel de peligro y del nivel de vulnerabilidad. Evaluación del peligro o amenaza, dependiendo del tipo de fenómeno subyacente (Gráfico N²), si son causados por fenómenos naturales o humanos (antrópicos o antropogénicos). Para cada fenómeno se establecen los criterios de evaluación; por ejemplo, para el peligro inundaciones generados por fenómenos hidrometeorológicos, los criterios serán de dos tipos: los propios del fenómeno (magnitud, duración y frecuencia de las lluvias) y los relacionados con las condiciones del lugar (quebradas, altas pendientes y vegetación) que agravan la manifestación del fenómeno. Evaluar la vulnerabilidad es medir el grado de exposición, fragilidad y resiliencia de los elementos dispuestos en el lugar de ocurrencia del fenómeno (viviendas, edificaciones, personas, ganado, obras, etc.).

\section{Gráfico $\mathrm{N}^{\circ}$ 1: Esquema general de la gestión del riesgo de desastres}

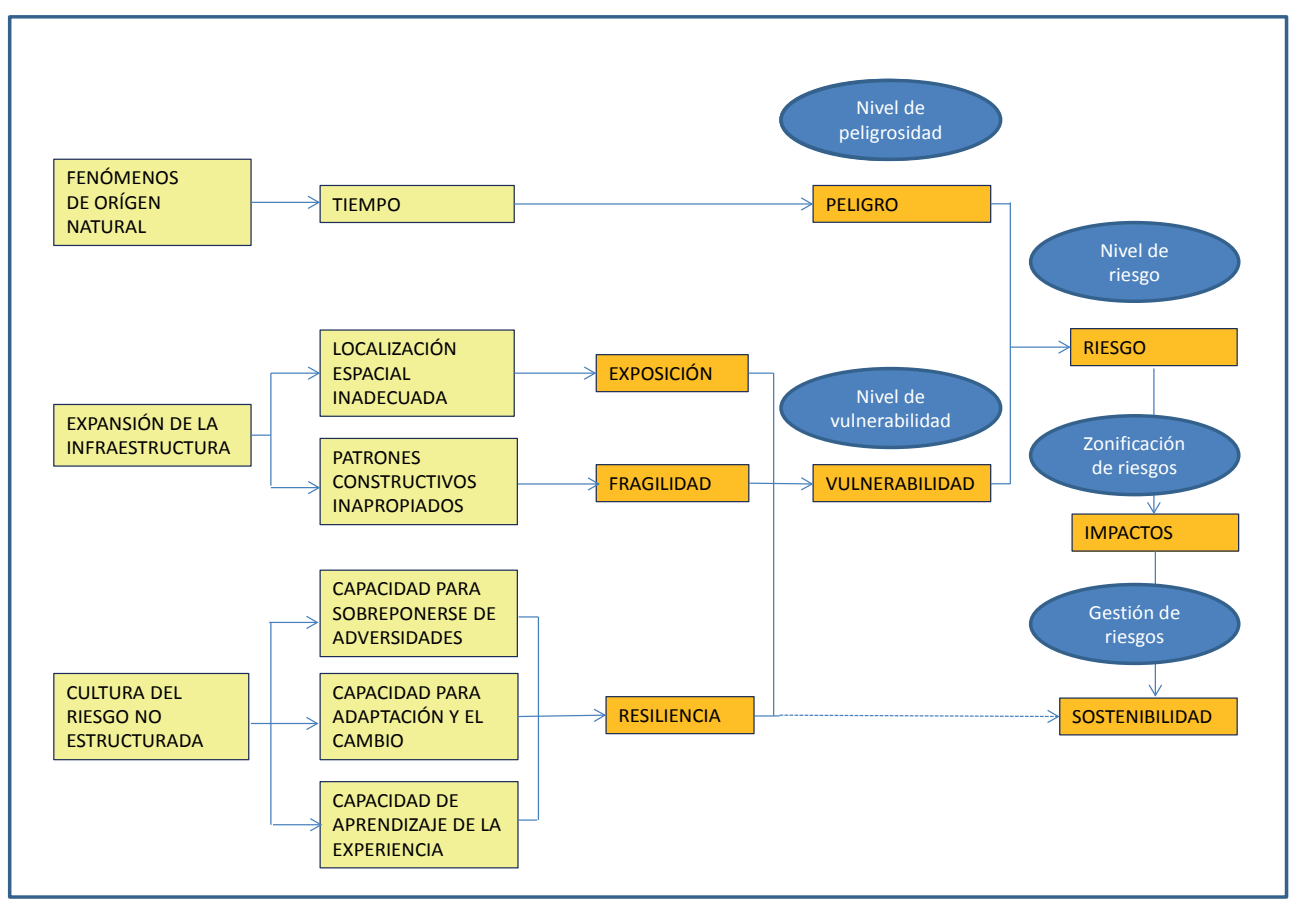




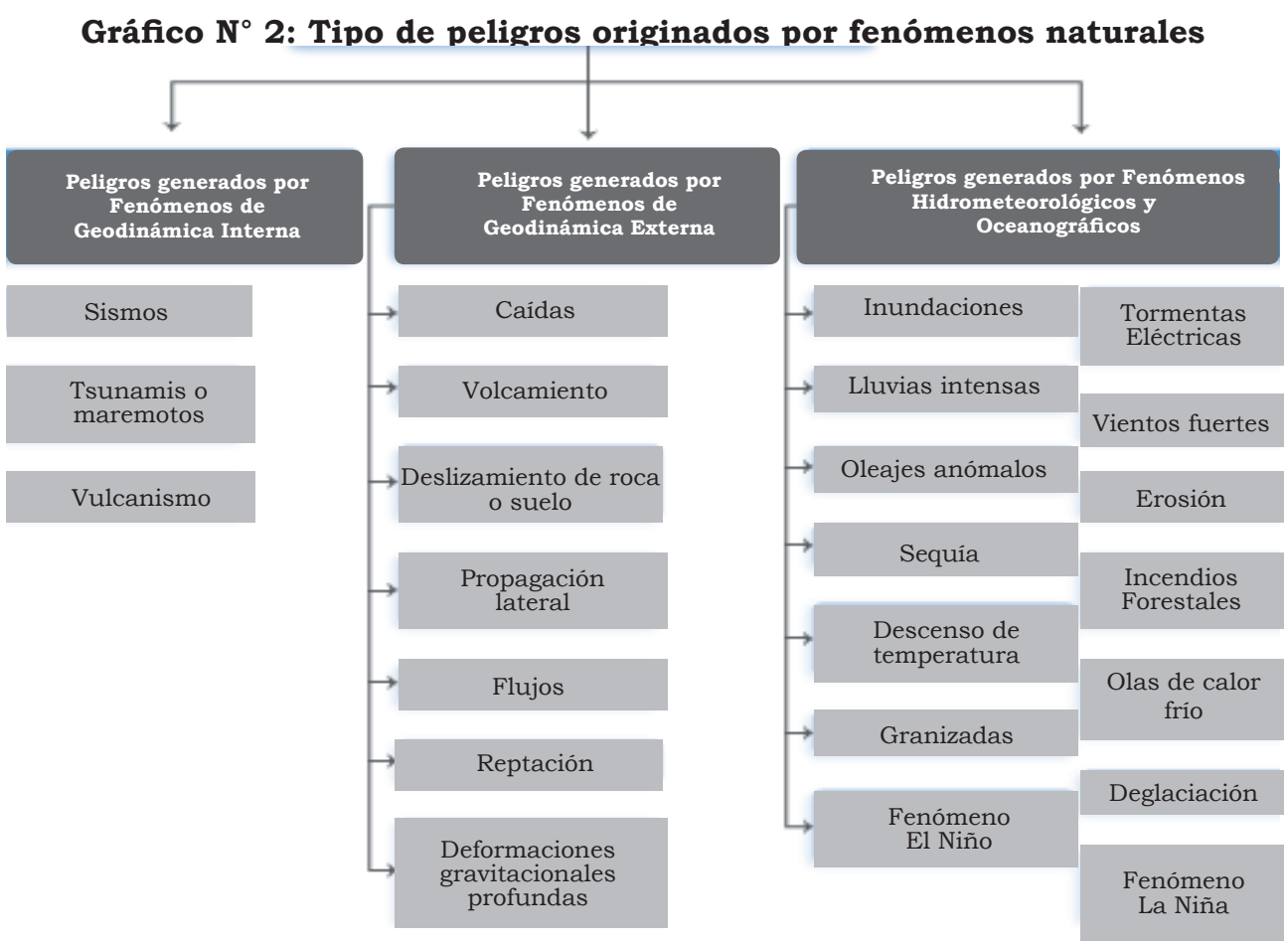

Estimado el riesgo de desastres, este se grafica en un mapa de riesgo, el que zonifica los niveles de riesgo por cada zona. Se establecen los puntos críticos, por ejemplo, lugares donde el río se puede desbordar, quebradas por donde discurrirá el torrente de agua con sus arrastres de lodo, piedras, troncos, o laderas de cerros con viviendas. Consecuentemente, se realiza la gestión del riesgo de desastres con el establecimiento de dos tipos de medidas de control: estructurales y no estructurales. Las medidas estructurales consisten en construcción física para reducir o evitar los riesgos, o aplicar técnicas de ingeniería para lograr la resistencia y resiliencia de las estructuras o sistemas físicos frente a los peligros. Generalmente están orientados a controlar u orientar una masa hídrica (cauce, agua de escurrimiento) para reducir las inundaciones. Las medidas no estructurales comprenden cualquier medida que no suponga una construcción física y que utiliza el conocimiento, las prácticas o los acuerdos existentes para prevenir o reducir el riesgo y sus impactos, especialmente a través de leyes, políticas, conciencia pública, educación y capacitación. En general, no intenta aumentar la capacidad de un cauce, sino minimizar los daños a la población. Una medida no estructural, como un sistema de alerta temprana (SAT), puede implicar una construcción física accesoria, por ejemplo, una caseta para instalar pluviógrafos. Pero esta construcción no intenta modificar 
las condiciones del flujo sobre el cauce, sino, más bien, aumentar la información y la disponibilidad oportuna de ésta por la población.

De esta forma, la gestión del riesgo de desastres se convierte en un proceso que contribuye decisivamente al desarrollo sostenible de una sociedad.

\subsection{La interrupción del desarrollo}

El análisis realizado por la Corporación Andina de Fomento (CAF) revela que los daños totales originados por el Fenómeno El Niño 1997-98 en el Perú ascienden a los 3.500 millones de dólares. Ello incluye daños directos por valor de 1.612 millones de dólares (el 46\% del total), y daños o pérdidas indirectas, por 1.888 millones adicionales (el 54\%). Ver Cuadro $\mathrm{N}^{\circ} 4$ (CAF, 1999)

Los daños directos ocasionados por los desastres, son las pérdidas del stock de bienes así como los costos en que será necesario incurrir para reponer los acervos de capital en su estado anterior al desastre. Los daños indirectos se refieren a los mayores gastos en que ha sido necesario incurrir y los menores ingresos que se han percibido en la prestación de determinados servicios, así como a la producción agropecuaria que se haya dejado de obtener como resultado de los daños directos. Un ejemplo ilustrativo de ambos tipos de daños se presenta cuando una plantación de uva sufre una severa inundación. Las pérdidas directas es el valor de la inversión en plantones, de instalaciones para acondicionar las parras y gastos como mano de obra, fumigación, fertilización, poda, etc., así como también de los equipos de riego tecnificado. Las pérdidas indirectas estarian representadas por el valor de las cosechas que se dejarian de tener como ingreso, más costos de financiamiento. 
CUADRO N 4: DAÑOS POR SECTORES DEL FENÓMENO EL NIÑO 1997-1998

\begin{tabular}{|c|c|c|c|c|}
\hline SECTOR Y SUBSECTOR & $\begin{array}{c}\text { DAÑO TOTAL } \\
\text { MILLONES DE USS }\end{array}$ & $\begin{array}{l}\text { DAÑO DIRECTO } \\
\text { MILLONES DE USS }\end{array}$ & $\begin{array}{l}\text { DANO INDIRECTO } \\
\text { MILLONES DEUSS } \\
\end{array}$ & $\begin{array}{c}\text { EFECTO EN LA } \\
\text { BALANZA DE PAGOS }\end{array}$ \\
\hline SECTORES SOCIALES & 485 & 457 & 28 & 81 \\
\hline VIVIENDA & 223 & 202 & 21 & 33 \\
\hline EDUCACIÓN & 228 & 224 & 4 & 36 \\
\hline SALUD & 34 & 31 & 3 & 12 \\
\hline SECTORES DE SERVICIOS & 237 & 174 & 63 & 116 \\
\hline AGUA Y SANEAMIENTO & 71 & 63 & 8 & 24 \\
\hline ELECTRICIDAD & 166 & 111 & 55 & 92 \\
\hline SECTOR TRANSPORTE & 717 & 604 & 113 & 248 \\
\hline CARRETERAS & 686 & 572 & 113 & 248 \\
\hline FEROCARRILES & 31 & 31 & 0 & 0 \\
\hline SECTORES PRODUCTIVOS & 1,627 & 319 & 1,308 & 776 \\
\hline AGROPECUARIO & 613 & 163 & 550 & 225 \\
\hline PESCA & 26 & 15 & 11 & 3 \\
\hline MINERÍA & 44 & 28 & 16 & 10 \\
\hline INDUSTRIA & 675 & 7 & 668 & 538 \\
\hline COMERCIO & 269 & 106 & 163 & 0 \\
\hline OTROS DAÑOS & 434 & 58 & 376 & 162 \\
\hline INFRAESTRUC. DE GOBIERNO & 58 & 58 & 0 & 0 \\
\hline GASTOS DE PREVENCIÓN & 219 & 0 & 219 & 0 \\
\hline GASTOS DE EMERGENCIA & 157 & 0 & 157 & 0 \\
\hline TOTAL NACIONAL & 3,500 & 1,612 & 1,888 & 1,383 \\
\hline
\end{tabular}

Estimación de la CAF

Los sectores productivos fueron los más afectados con el 46.5\% del total de las pérdidas. En segundo lugar, el sector transporte representó el 20.5\%. Si bien se nota que entre los sectores productivos las mayores pérdidas sucedieron en el sector industria, con $19.3 \%$, seguido por la agricultura, con el $17.5 \%$, habría que considerar que parte de la industria estropeada es agroindustria, con lo que el sector agricultura sería el sector más afectado. En la misma línea de pensamiento, el perjuicio en el sector transporte provocaría mayores daños a la agricultura, porque esta actividad se encuentra muy difundida en el territorio y el comercio pasa por la concentración de la producción utilizando el transporte de carga para conducirla a mercados, almacenes, agroindustria o puntos de transbordo. En la misma lógica, parte importante de las pérdidas en el sector comercio estaría representado por los servicios de este sector a la agricultura, tales como certificación, procesamiento, envases, impresiones, publicidad, entre muchos otros. Incluso, de los gastos en prevención, parte importante se orienta a descolmatar lechos de río, construir diques, represas, muros de contención, entre obras diversas, con lo que se acrecienta el impacto en la deseconomía de la agricultura. De igual forma, habria que sumar los gastos en atención de emer- 
gencias efectuados en el agro.

A lo anterior, considerariamos como efectos indirectos adicionales el detrimento en el sector externo. Por un lado, las divisas que se dejarian de obtener al dejar de exportar y, por el otro, las divisas que se emplearian en importar los productos para mantener el equilibrio del mercado. En la última columna del Cuadro $\mathrm{N}^{\circ} 6$ se estiman estos efectos adversos, tocándole al sector agropecuario el $16.3 \%$ del deterioro total en la balanza de pagos.

También se tiene en cuenta el impacto del Fenómeno El Niño en el crecimiento sectorial y global de la economía peruana, la reducción de la inversión para expandir la capacidad productiva del país en la misma medida del gasto para reconstruir los activos perdidos; es decir, se invierte para tener el mismo nivel anterior al desastre y no para agrandar la base productiva. Igualmente, la menor producción conlleva a una inflación manifestada en los precios de los productos agrarios. Entre todas las áreas más afectadas por el Fenómeno El Niño, la costa norte y central es la que más sufrió la embestida, justamente donde se encuentra concentrada la mayor parte de la población del país y, además, los predios de cultivos exportables.

Teniendo una agricultura predominante de secano (agua de lluvia), que abarca el $63.8 \%$ de la superficie agrícola nacional, y que el $31.8 \%$ es de riego por gravedad (agua distribuida por canales), consolida el $95.6 \%$ de la superficie agrícola habilitada con agua de superficie; lo cual revela que solo el
4.4\% de las tierras para uso agrícola aplica el riego tecnificado. Por tanto, si el Fenómeno El Niño exacerba la masa hídrica superficial, entonces, la agricultura se encuentra notoriamente expuesta a un mayor riesgo de desastre.

En efecto, el exceso de agua de superficie se desplaza en forma conducida por cursos de río, y la no conducida, que es el agua de escorrentía, la cual normalmente se infiltra en el suelo, pero que al ser abundante corre con fuerza destructiva sobre los terrenos, en proporción directa a la pendiente, naturaleza del suelo y cobertura vegetal. Ella arrasa viviendas, ganado, produce huaycos, erosiona el suelo, inunda cultivos, colmata cursos de agua, destruye caminos, debilita puentes, obstruye con lodo el alcantarillado, etc.

La alta temperatura correlacionada al Fenómeno El Niño, produce el fenómeno de tropicalización. Temperatura y humedad tienen una elevada incidencia en el aumento de plagas y enfermedades en los cultivos, produciendo una merma sustantiva por aspectos sanitarios en las cosechas; como también por afectar el proceso biológico, afectando la floración y la formación de tubérculos; el estrés calórico en los animales repercute en la mortalidad, morbilidad y baja de rendimiento.

Resumiendo, tenemos que el impacto del Fenómeno El Niño 19971998, causó daños a la economía valorizados en 3,500 millones de US\$. En el año 1998, el valor total de las exportaciones peruanas fue de 5,757 
millones de US\$, lo que representó el $60.8 \%$ del total exportado, tanto de productos tradicionales como no tradicionales. Concentrándonos en el sector agropecuario, las pérdidas ascendieron a 613 millones de US\$, los que relativizados a los 625 millones de US\$, simbolizan el 98.1\%; lo equivale a expresar que las pérdidas en el sector agropecuario casi tienen el mismo valor de las exportaciones en ese año.

Es tácito que se ha abarcado solo un fenómeno y queda como reflexión el análisis de los impactos de otros fenómenos: sequías, heladas, granizadas y desglaciación. Pero hay que considerar que el Fenómeno El Niño es uno de los principales por sus impactos, pero a la vez, plantea mayores posibilidades de ser mitigado.

En suma, el riesgo de desastres en el Perú tiene una magna relevancia para la agricultura por sus periódicos impactos desfavorables, que pueden significar desde un ligero desvanecimiento hasta un colapso drástico con repercusiones severas en las unidades productivas y en su lograda competitividad, lo que podría significar una recuperación que puede tardar varios años.

\section{LA GESTIÓN DEL RIESGO DE DESASTRES}

\subsection{Normatividad básica}

El 22 de enero del 2005 culminó la Conferencia Mundial sobre la Reducción de los Desastres en Kobe, Hyogo, Japón, aprobándose el "Marco de Ac- ción de Hyogo 2005-2015: Aumento de la Resiliencia de las Naciones y Comunidades ante los Desastres". En él se propugna que cada país debe garantizar que el enfoque de reducción del riesgo de desastres (RRD) sea una prioridad nacional y local, y tenga una sólida base institucional para su implementación.

El 17 de diciembre del 2010, el Acuerdo Nacional amplía el acta suscrita el 22 de julio del 2002, para incorporar la trigésima segunda política de Estado, Gestión del Riesgo de Desastres, la cual debe ser implementada por los organismos públicos de todos los niveles de gobierno, con la participación activa de la sociedad civil y la cooperación internacional, promoviendo una cultura de la prevención y contribuyendo directamente en el proceso de desarrollo sostenible a nivel nacional, regional y local.

El 08 de febrero del 2011 se promulga la Ley $\mathbf{N}^{\circ} \mathbf{2 9 6 6 4}$, con la que se crea el Sistema Nacional de Gestión del Riesgo de Desastres-SINAGERD, y el 25 de mayo del 2011 se aprueba el Reglamento de la Ley, mediante Decreto Supremo N ${ }^{\circ}$ 048-2011-PCM.

El 13 de abril del 2012 se aprobó la R.M. N088-2012-PCM: Lineamientos Técnicos Generales para Implementación del Proceso de Estimación del Riesgo, constituido por procedimientos técnicos y administrativos que orientan uno de los procesos que corresponden a la Gestión del Riesgo de Desastres. Mediante ellos, se busca confiabilidad, oportunidad y utilidad de la información relativa al conoci- 
miento de los peligros, vulnerabilidades y sustento de los niveles de riesgo que permita tomar las decisiones más apropiadas y efectivas de gestión del riesgo de los desastres.

El 29 de mayo del 2012 se publica la Ley N²9869: Ley de Reasentamiento Poblacional para Zonas de Muy Alto Riesgo no Mitigable, con el fin de proteger la vida, el equipamiento y los servicios, dirigido a los centros poblados y población dispersa ubicada en lugares cuya vulnerabilidad es tan grande que impide la opción de habitabilidad.

El 24 de octubre del 2012 se aprueba, mediante R.M. N²76-2012-PCM, los Lineamientos para la Constitución y Funcionamiento de los Grupos de Trabajo de la Gestión del Riesgo de Desastres en los tres niveles de gobierno, los que apuntan a guiar la gestión de los Grupos de Trabajo establecidos por la Ley del SINAGERD, que son las instancias de mayor decisión en cada órgano de gobierno.

E1 02 de noviembre del 2012, se aprueba el Decreto Supremo $N^{\circ} 111$ 2012-PC: Politica Nacional de Gestión del Riesgo de Desastres, que incorpora a la Gestión del Riesgo de Desastres como una Política Nacional de cumplimiento obligatorio por todos los niveles del gobierno nacional. Comprende un conjunto de orientaciones dirigidas a impedir o reducir los riesgos de desastres, evitar la generación de nuevos riesgos y efectuar una adecuada preparación, atención, rehabilitación y reconstrucción ante situaciones de desastres, así como minimizar sus efectos adversos sobre la población, la economía y el ambiente.

El 12 de mayo del 2014 se aprueba el Decreto Supremo N`34-2014-PCM: Plan Nacional de Gestión del Riesgo de Desastres 2014-2021 (PLANAGERD), el que establece un objetivo nacional, seis objetivos estratégicos, catorce objetivos específicos y 47 acciones estratégicas, los que deberán ser tomados en cuenta por todas las entidades públicas para implementar sus metas concretas e indicadores de desempeño.

\subsection{Responsabilidades de entidades públicas}

Todas las entidades públicas, de los gobiernos nacional, regional y local, deben alinearse al Plan Nacional de Gestión del Riesgo de Desastres. En tal sentido, el Art. $39^{\circ}$ del Reglamento de la Ley $\mathrm{N}^{\circ} 29664$, expresa: "En concordancia con el Plan Nacional de Gestión del Riesgo de Desastres, las entidades públicas en todos los niveles de gobierno formulan, aprueban y ejecutan, entre otros, los siguientes Planes:

- Planes de Prevención y Reducción del Riesgo de Desastres.

- Planes de Preparación.

- Planes de Operaciones de Emergencia.

- Planes de Educación Comunitaria.

- Planes de Rehabilitación.

- Planes de Contingencia.

La autoridad correspondiente con- 
La gestión del riesgo de desastres en el Perú

vocará a las entidades privadas y a la sociedad para el desarrollo de estos instrumentos de planificación".

En los planes deben estar los siete procesos técnicos de las gestión del riesgo de desastres:

Estimación del riesgo: genera conocimiento de peligros y vulnerabilidades.

Prevención del riesgo: acciones que evitan nuevos riesgos.

Reducción del riesgo: reducir vulnerabilidades y riesgos existentes.

Reconstrucción: establecer sostenibilidad sin riesgo de áreas afectadas.

Preparación: adecuación organizacional para un peligro o desastre inminente.

Respuesta: atención de emergencia o desastre después de ocurrido.

Rehabilitación: restablecer servicios básicos e inicio de reparación de daños.

Los cuatro primeros procesos están a cargo del Centro Nacional de Estimación, Prevención y Reducción del Riesgo de Desastre (CENEPRED), creada con la La Ley $\mathrm{N}^{\circ} 29664$. Los tres restantes son responsabilidad del Instituto Nacional de Defensa Civil (INDECI), entidad preexistente.

Mediante los planes, todos los organismos públicos, organizaciones privadas y la población deben analizar los riesgos de desastres y exponer y disponer sus prioridades, energía y recursos para evitarlos o reducirlos.

El total de organismos de gobierno público ascienden a 1885 , cifra que consolida a:
- 18 ministerios.

- 25 gobiernos regionales.

- 1842 gobiernos locales

\subsection{Perfil de la gestión en riesgo de desastres}

Una evaluación hecha en el 2015, a cinco años de explicitada la voluntad del Estado, mediante la Ley $N^{\circ} 29664$, para conducir el desarrollo del país sin el quebranto de los desastres, examinaremos someramente cómo están actuando los órganos responsables de conducir los procesos técnicos de la gestión del riesgo de desastres en sus propios ámbitos. Ver Cuadro $\mathrm{N}^{\circ} 5$.

Para tal efecto, tomaremos información del propio CENEPRED, en su informe de seguimiento a junio del 2015, centrándonos solo en diez indicadores:

- Constitución del Grupo de Trabajo: Es proyectar la resolución de alcaldía, en la que la máxima autoridad de cada ente de gobierno con los funcionarios jefes de órganos deben constituir el Grupo de Trabajo de la Gestión del Riesgo de Desastres (GTGRD).

- Instalación del Grupo de Trabajo: Es tener el acta de la primera reunión del GTGRD.

- Formulación de Plan de Trabajo: Es aprobar el Plan de Trabajo del GTGRD.

- Formulación de Reglamento Interno: Es aprobar el Reglamento Interno de Funcionamiento del GTGRD.

- Capacitación y asistencia técnica: 
Si el GTGRD o funcionarios han sido fortalecidos en sus capacidades.

- Actualización del ROF: Si se ha modificado el Reglamento de Organización y Funciones de la organización para incorporar como responsabilidad la gestión del riesgo de desastres.

- Formulación de un Plan en GRD: Si cuentan con el Plan de Prevención y Reducción del Riesgo de
Desastres.

- Mecanismos de monitoreo: Si tienen dicho mecanismo.

- Aplican al PP 068: Si han accedido al Programa Presupuestal 068, que es una fuente de financiamiento específica para gestión del riesgo de desastres.

- Conocen el PLANAGERD: Si tienen conocimiento del Plan Nacional de Gestión del Riesgo de Desastres: 2014-2021.

\section{CUADRO N 5: INDICADORES DE SEGUIMIENTO A JUNIO DEL 2015}

\begin{tabular}{|c|c|c|c|c|c|}
\hline \multirow[t]{2}{*}{ INDICADORES } & MINISTERIOS & $\begin{array}{r}\text { GOBIERNOS } \\
\text { REGIONALES } \\
\end{array}$ & \begin{tabular}{|c|} 
MUNICIPALIDADES \\
PROVINCIALES \\
\end{tabular} & $\begin{array}{l}\text { DISTRRTOS } \\
\text { LIMENOS } \\
\end{array}$ & $\begin{array}{c}\text { OTROS } \\
\text { DISTRMOS } \\
\end{array}$ \\
\hline & 18 & 25 & 196 & 49 & 1597 \\
\hline Constitución del Grupo de Trabajo & 100.0 & 100.0 & 49.0 & 85.7 & 8.9 \\
\hline Instalación del Grupo de Trabajo & 77.8 & 92.0 & 37.8 & 67.3 & 6.8 \\
\hline Formulación Plan de Trabajo & 61.1 & 36.0 & 19.9 & 40.8 & 3.4 \\
\hline Formulación del Reglamento Intemo & 66.7 & 32.0 & 14.8 & 44.9 & 1.9 \\
\hline Actualización del ROF & 38.9 & 12.0 & 9.7 & 34.7 & 0.7 \\
\hline Capacitación y asisten cia técnica & 16.7 & 16.0 & 10.7 & 20.4 & 0.2 \\
\hline Formulación del Plan GRD & 11.1 & 28.0 & 9.2 & 28.6 & 0.9 \\
\hline Mecanismos de monitoreo & 11.1 & 24.0 & 5.1 & 16.3 & 0.6 \\
\hline Aplican al PP 068 & 50.0 & 76.0 & 25.0 & 46.9 & 3.4 \\
\hline Conocen el PLANAGE RD & 27.8 & 20.0 & 7.1 & 18.4 & 0.3 \\
\hline
\end{tabular}

Fuente: CENEPRED-DIMSE. Informe de Monitoreo, Seguimiento y Evaluación de la Política y Plan Nacional de Gestión del Riesgo de Desastres: Gestión Prospectiva y Reactiva. Setiembre del 2015. Elaboración propia.

El indicador Constitución del Grupo de Trabajo encarna la primera acción que debe realizar un ente de gobierno para dar una seña de cumplimiento de la Ley. Se cumple este requisito proyectando una resolución de la autoridad (resolución ministerial, regional o local), en el que señala las autoridades de alto nivel que la conforman bajo la presidencia de la máxi- ma autoridad (Ministro, presidente del gobierno regional o alcalde). Es un requisito simple, que ha sido cumplido por la totalidad de los ministerios y gobiernos regionales, casi las dos terceras partes de los distritos de la capital, la mitad de las municipalidades provinciales y menos de un décimo de las municipalidades distritales, exceptuando las de Lima Metropolitana. El 
grado de cumplimiento de este indicador es un marcador importante de la omisión total a los mandatos legales.

La Instalación del Grupo de Trabajo, es la acción inicial de funcionamiento del grupo de trabajo. Se concreta con la primera reunión del grupo de trabajo, la cual se registra en actas y se toman los primeros acuerdos. Se refleja que en todos los casos hay entidades públicas que dieron el primer paso formal, pero nunca se han reunido.

Formulación del Plan de Trabajo del Grupo de Trabajo y Formulación del Reglamento Interno de funcionamiento del Grupo de Trabajo constituyen dos indicadores que reflejan la proyección de la acción de cada Grupo de Trabajo. Los avances son menores en todos los niveles de gobierno.

Los cuatro primeros indicadores están referidos al Grupo de Trabajo, el cual es un espacio de coordinación y de decisión, intra e interinstitucional, en forma indelegable. El cuarto indicador es el que refleja el grado de formalización de la entidad en cuanto al funcionamiento del Grupo de Trabajo. La situación es la siguiente: el $66.7 \%$ de los ministerios, 32\% de las regiones, $14.8 \%$ de los municipios provinciales, $44.9 \%$ de los distritos limeños y el $1.9 \%$ de demás distritos del país se puede decir que disponen de un grupo de trabajo con signos de funcionamiento.

El siguiente indicador extiende la responsabilidad de la gestión del riesgo de desastres (GRD) a todos los órganos de cada gobierno. Se trata de modificar o actualizar el Reglamento de Organización y Funciones de la entidad de gobierno, en el que aparezcan funciones relacionadas al tema de la GRD, toda vez de las leyes orgánicas de los gobiernos (nacional, regional y local) son anteriores a la Ley $\mathrm{N}^{\circ} 29664$. Una vez incluida las funciones, las acciones y el uso de los recursos adquieren legitimidad.

El indicador Capacitación y Asistencia Técnica grafica palmariamente los bajos niveles de fortalecimiento de capacidades recibidos: $16.7 \%$ de los ministerios, $16 \%$ de las regiones, $10.7 \%$ de los municipios provinciales, $20.4 \%$ de los distritos limeños y $0.2 \%$ de los demás distritos. Estos avances explican en cierta forma el bajo adelanto en la aplicación de la GRD en el país.

El siguiente indicador, Formulación de un Plan en Gestión del Riesgo de Desastres, es clave para determinar si en la región han explicitado el qué hacer en el tema de la GRD. Puede notarse que gradualmente los distintos ámbitos de gobierno van disminuyendo en la aplicación de la norma. Los grados de cumplimiento, son: ministerios $11.1 \%$, regiones $28 \%$, municipalidades provinciales $9.2 \%$, municipios distritales limeños 28.6\%, y municipios del resto del país 0.9\%.

El indicador Mecanismos de Monitoreo precisa si se examina la marcha del Plan de la GRD y se propicia la mejor ejecución. Todas las mediciones son menores que en el indicador anterior. Ministerios $11.1 \%$, regiones $24 \%$, municipalidades provinciales $5.1 \%$, 
municipios distritales limeños 16.3\% y municipios del resto del país $0.6 \%$. Este indicador es sintesis de la capacidad de gestión máxima en riesgo de desastres en cada nivel de gobierno. Obviamente, referido a la etapa de crear condiciones para su aplicación; ya que el informe del CENEPRED no mide indicadores asociados a la ejecución de acciones concretas, ejecución presupuestal, inversión en GRD, etc. Si se tratara de estos logros, el avance sería desasosegador.

El Programa Presupuestal 068: Reducción de la Vulnerabilidad y Atención de Emergencia (PREVAED) está orientado a financiar fundamentalmente la capacitación y asistencia técnica, seguridad de locales escolares $\mathrm{y}$ asistencia a poblaciones en emergencia y desastre, principalmente educativas. El avance en este indicador está liderado por acciones del sector educación en toda la república.

El último indicador: Conocen el Plan Nacional de Gestión del Riesgo de Desastres (PLANAGERD), es un indicador que muestra la gravedad del Sistema de Gestión del Riesgo de Desastres, toda vez que el grado de conocimiento es solo eso, saber de su existencia. No implica haber desarrollado planes en GRD para sus respectivos ámbitos que estén consistenciados con el PLANAGERD o la programación de metas específicas contributivas a las metas del PLANAGERD. E1 27.8\% de los ministerios, $20 \%$ de las regiones, $7.1 \%$ de los municipios provinciales, $18.4 \%$ de los distritos limeños, y el $0.3 \%$ de demás distritos del país conocen el PLANAGERD.

La evaluación de los diez indicadores analizados deja el interrogante de, si con el grado de avance en las condiciones que la institucionalidad del SINAGERD presenta en la actualidad, ¿se podrían lograr las metas propuestas por el PLANAGERD y así contribuir a dar seguridad a la vida y el entorno de todos los pueblos del país?

\section{CONCLUSIONES Y RECOMENDA- CIONES}

\subsection{Conclusiones}

a) La historia de los desastres en el Perú da cuenta de una vasta colección de hechos que parecen inmortales. Entre todos ellos, los peligros de tipo climatológicos (lluvias intensas, inundaciones, heladas y huaycos) tienen una alta incidencia y asombran por los daños que originan, y la conmoción que causan a la economía, a la sociedad y al ambiente nacional. Entre todos los sectores, es en la agricultura, donde los daños causados por los desastres en el Perú tienen un impacto negativo muy severo. Esto se constata fácilmente por cuanto esta actividad se caracteriza por utilizar ampliamente el recurso suelo y el clima. En tal sentido, las condiciones ambientales cuando se extralimitan (abundancia), o son muy escasas (sequias), u ocurren en forma inoportuna, afectan 
drásticamente los cultivos y las crianzas, el suelo, la distribución del agua, y provocan la presencia de plagas y enfermedades. Por tanto, la agricultura se ve lastimada y dentro de ella el sector moderno de exportación. El Fenómeno El Niño de 19971998, cuyos mayores impactos fueron tangibles en los primeros meses de 1998, vulneraron la economía en un valor equivalente al $60.8 \%$ del total exportado; $\mathrm{y}$, específicamente, las pérdidas en el sector agropecuario, significaron el $98.1 \%$ de lo que dicho sector exportó. Entonces, desde este punto de vista, el año 1998 fue un año casi totalmente improductivo para la agricultura peruana.

b) El Estado peruano ha establecido, desde el 2011, una nueva institucionalidad que ha desplazado la concepción antigua de los desastres (concepción reactiva), a la nueva concepción de la gestión del riesgo de los desastres (concepción preventiva). Es decir, los desastres se pueden evitar o reducir. Para tal efecto, el SINAGERD ha implantado normas e instrumentos para instalar capacidades de gestionar los riesgos de desastres en todas las entidades públicas. Si bien la voluntad del Estado peruano ha quedado manifiesta, la realidad es que los avances en la nueva institucionalidad, categó- ricamente, demuestran un débil adelanto en las capacidades de gestionar el riesgo de los desastres en el sector público. Así, los niveles de gobierno no han dispuesto de un mecanismo de monitoreo como parte de su gestión en niveles muy altos: Ministerios $88.9 \%$, regiones $76 \%$, municipalidades provinciales 94.9\%, municipios distritales limeños $83.7 \%$, y municipios del resto del país $99.4 \%$. Visto desde otra perspectiva, el $72.2 \%$ de los ministerios, $80 \%$ de las regiones, 92.9\% de los municipios provinciales, $81.6 \%$ de los distritos limeños, y el 99.37\% de los demás distritos del país desconocen el PLANAGERD. Si lo desconocen ¿qué papel juegan en su implementación? y, ¿no será también significativo el desconocimiento de otras normas e instrumentos técnicos elaborados por los entes rectores del SINAGERD?

c) Se configura una situación de poco aliento. Grave a nivel sectorial (18), regional (25), provincial (196) y en los distritos limeños (49), porque están a la mano $\mathrm{y}$ son las entidades que cuentan con mayores recursos y mejores especialistas. Muy grave en los demás distritos $(1,597)$, porque tienen menores recursos, especialistas de todo tipo y por el bajísimo acceso a la capacitación y la asistencia técnica.

d) Considerando que el SINAGERD es un sistema que debe globali- 
zar al sector privado y toda la población, justamente, donde está la mayor proporción de damnificados y afectados por los desastres, se colige el poco arrastre del SINAGERD a los verdaderos destinatarios del servicio de dar seguridad ante el riesgo de los desastres.

e) A los cinco años de la dación de la Ley $\mathrm{N}^{\circ}$ 29664, la proyección es preocupante. ¿Cuánto tiempo ha de pasar para llegar a instituir una eficaz gestión del riesgo de los desastres, al menos en la quinta parte de los distritos del interior del país? La existencia de un cuerpo importante de organismos de gobierno que no están consolidados en la gestión del riesgo expone la debilidad del SINAGERD, toda vez que el concepto de sistema abarca la totalidad y no parte de ella. No hay un sistema de una parte. Es decir, un sistema trasciende 10 conceptual cuando incorpora en la práctica a todos los elementos constitutivos y, entre ellos, se manifiesta una interdependencia, todo en aras del propósito de proteger la vida o la calidad de esta.

f) A manera de corolario, todo indica que, en los próximos años, la gestión del riesgo de los desastres en el país seguirá siendo insuficiente y, por ende, la agricultura seguirá interrumpida en su desarrollo. Esta situación implicará pérdida de activos, de mer- cados y una disminución importante de la competitividad de los agentes agrarios y, consiguientemente, una merma sensible de la sostenibilidad. Tácitamente, por razones de contexto y no por cuestiones de debilidad de las unidades empresariales. En definitiva, un desplazamiento de la competitividad a la calamidad en el agro.

\subsection{Recomendaciones}

a) Reforzar las entidades conformantes del SINAGERD, fundamentalmente el CENEPRED. Dentro de este afianzamiento, el órgano de fortalecimiento de capacidades debería merecer excepcional atención, toda vez, que es el facilitador de capacidades en gestión del riesgo de desastres de toda la institucionalidad pública. Del CENEPRED se han originado importantes normas y es incuestionable su capacidad para normar científica y técnicamente los procesos de la GRD; sin embargo, no muestra la misma fortaleza en hacer accesible y aplicable dichas normas a los órganos responsables de ejecutarlas.

b) La estrategia para expandir el SINAGERD debería basarse en el acompañamiento de socios sinérgicos, como ENIEX, ONG's, universidades y consultores especializados. En especial, las entidades que tienen una con- 
tribución apreciable en el campo de la gestión del riesgo de desastres, como Soluciones Prácticas y PREDES.

c) Sería conveniente revisar la estrategia de formular los planes de prevención y reducción del riesgo de los desastres, la que debería estar basada en la participación de la población y del sector privado. El empoderamiento de este sector no público sería importante por ser el objeto y sujeto de toda acción concebida por el SINAGERD. Aquí les tocaría un papel importante a las empresas del agro, toda vez que es imperativo mantener su competitividad y sostenibilidad.

d) Las prioridades deberian estar en función de la prospección de daños por cuencas, lo cual conllevaria a realizar evaluacio- nes del riesgo; ulteriormente, los planes respectivos, que con la debida antelación puedan definirse y gestionar proyectos, financiamiento y actividades preventivas y de reducción del riesgo, como también sistemas de alerta temprana. La culminación de todo este esfuerzo sería lograr la sostenibilidad del agro nacional $\mathrm{y}$, colateralmente, de otros sectores de actividad.

e) Es imperiosa la necesidad de apretar el acelerador para la implantación de los procesos de la gestión del riesgo de desastres, sobre todo los de estimación, la prevención y reducción del riesgo. En los años inmediatos se debe resolver la amplia brecha de organismos de gobierno que, en la práctica, no están integrados al SINAGERD. 


\section{REFERENCIAS BIBLIOGRÁFICAS}

CAF (1999). El Fenómeno El Niño 1997-1998: Memoria, Retos y Soluciones. Volumen V: Perú.

CENEPRED (2015). Informe de Monitoreo, Seguimiento y Evaluación de la Política y Plan Nacional de Gestión del Riesgo de Desastres: Gestión Prospectiva y Reactiva. Lima, 30 de Setiembre del 2015. http://cenepred.gob.pe/dimse/Informe_II_Trimestre_2015.pdf.

CENEPRED (2015). Manual para la Evaluación de Riesgos originados por Fenómenos Naturales. Lima, segunda edición.

CONGRESO (2011). Ley N²9664: Ley del Sistema Nacional de Gestión del Riesgo de Desastres.

FERRADAS, Pedro (2015). La memoria es también provenir: Historia mundial de los desastres. Soluciones Prácticas, Lima, 2015.

INDECI (2017). Base Datos de Emergencias y Daños en el 2017.

Rescatado el 30 de octubre del 2017: http://sinpad.indeci.gob.pe/ReportServer/Pages/ReportViewer.aspx?\%2fSINPAD\%2fEstadistica\%2fRpt_Esta_C3_ Variacion\&rs\%3aCommand=Render

INDECI (2017). Estadística de emergencia: Serie cronológica: 2003-2014. Rescatado el 30 de octubre del 2017: https://www.indeci.gob.pe/objetos/secciones $/ \mathrm{MTM}=/ \mathrm{NTM}=/$ lista $/ \mathrm{NDc0} / 201707251636461 . p d f$

ISLA ZEVALLOS, Arturo (2015). Guía Metodológica para Elaborar el Plan de Prevención y Reducción del Riesgo frente a Inundaciones Pluviales y Fluviales. Lima, Soluciones Prácticas.

SINPAD-INDECI (2017): Emergencias y Daños Producidos por el Niño Costero al 07 de agosto del 2017.

Rescatado el 30 de octubre del 2017: https://www.indeci.gob.pe/objetos/noticias $/ \mathrm{NTY}=/ \mathrm{NTE} 1 \mathrm{Mw}==/$ fil20170912123201.pdf 